\title{
Robust PARAFAC for incomplete data
}

\author{
Mia Hubert, Johan Van Kerckhoven†, Tim Verdonck ${ }^{\ddagger}$
}

April 12, 2012

\footnotetext{
${ }^{*}$ KU Leuven, Department of Mathematics - LStat, Celestijnenlaan 200B, BE-3001 Heverlee, Belgium

${ }^{\dagger}$ KU Leuven, Department of Mathematics - LStat, Celestijnenlaan 200B, BE-3001 Heverlee, Belgium

${ }^{\ddagger}$ KU Leuven, Department of Mathematics - LStat, Celestijnenlaan 200B, BE-3001 Heverlee, Belgium
} 


\begin{abstract}
Different methods exist to explore multi-way data. In this article we focus on the widely used PARAFAC (Parallel factor analysis) model, which expresses multi-way data in a more compact way without ignoring the underlying complex structure. An alternating least squares procedure is typically used to fit the PARAFAC model. It is however well known that least squares techniques are very sensitive to outliers and hence the PARAFAC model as a whole is a nonrobust method. Therefore Engelen and Hubert [2011] have proposed a robust alternative, which can deal with fully observed data, possibly contaminated by outlying samples. In this paper we present an approach to perform PARAFAC on data which contain both outlying cases and missing elements. A simulation study shows the good performance of our methodology. In particular we can apply our method on a dataset in which scattering is detected and replaced with missing values. This is illustrated on a real data example.
\end{abstract}

Keywords: multi-way, incomplete data, robustness, principal component analysis, expectation maximization. 


\section{Introduction}

The simultaneous measurement of variables gives rise to complex data structures, which can often be represented as multi-way data sets. For example in chemistry, fluorescence emission spectra measured at several excitation wavelengths for several samples, fluorescence lifetime measured at several excitation and emission wavelengths or any kind of spectrum measured chromatographically for several samples give rise to three-way data that can be organized in a cube. Note that one-way data and two-way data can respectively be arranged in vectors and matrices. Throughout this paper we will only consider three-way data, but most results can be generalized to data of higher order. An important advantage of using multi-way data instead of unfolding it into matrices is that a more adequate and interpretable model can be obtained. In this paper we will focus on the very popular decomposition method PARAFAC which can be seen as a generalization of principal component analysis (PCA) to higher order tensors [Smilde et al., 2004, Kroonenberg, 2008]. The sensitivity of PCA to outliers is well known and therefore various robust alternatives to it have been proposed in literature, see Hubert et al. [2008] for an overview. In standard multivariate problems (in which two-way data are examined) outliers are defined as observations that differ significantly from the majority of other samples. Recently, Engelen and Hubert [2011] have shown the tremendous influence of outlying samples on the classical alternating least squares PARAFAC algorithm [Smilde et al., 2004]. They have also proposed a robust version of this algorithm, 
which relies on the robust PCA method ROBPCA [Hubert et al., 2005]. In this setting, outliers are described as matrices that have a deviating profile compared towards the other ones. This robust PARAFAC method is also a very important component of the approach developed in Engelen et al. [2009] which can also handle scatter, which is a type of contamination that affects all samples.

A topic which has, however, not yet been discussed in the context of robust decomposing methods for multi-way data is how to deal with missing elements. Missing values are a common occurrence in the field of chemometrics and data can be missing due to different reasons, e.g. malfunction of one or more sensors, irregular measurement intervals between samples or different sampling frequencies for the various sensors. In what follows, we will mostly assume that the reason why a data point is missing is not related to its actual value, i.e. the data are missing at random (MAR) [Little and Rubin, 1987]. Missing completely at random (MCAR) is a stronger hypothesis, but we will assume that the data are at least MAR. A good method to deal with data containing missing elements is the expectation maximization (EM) algorithm [Dempster et al., 1977]. The EM algorithm basically consists of an iterative scheme where in each iteration two steps are carried out: (i) the missing elements are filled in by the values which they are expected to be (the expectation step or E-step) and (ii) the desired entity is estimated from the data in which missing elements have been filled in (called the maximization step or $M$-step if the estimates are obtained via maximum likelihood). Since the true values of the missing elements are unknown, the procedure is repeated 
until some convergence criterion is fulfilled. The EM algorithm has already been adapted to deal with missing data for the classical PARAFAC model [Bro, 1997, Tomasi and Bro, 2004], for classical PCA [Walczak and Massart, 2001], for robust estimation of the covariance matrix [Little, 1988, Cheng and Victoria-Feser, 2002], robust PCA [Serneels and Verdonck, 2008], robust PCR [Serneels and Verdonck, 2009] and robust PLS [Stanimirova et al., 2007]. In this article we investigate how the EM approach to deal with incomplete data can be extended to robust PARAFAC.

In Section 2 we explain the classical PARAFAC model and describe an algorithm to deal with missing values when fitting this model. The robust approach of Engelen and Hubert [2011] is summarized in Section 3 and in Section 4 we propose our approach to fit a PARAFAC model that can cope with outliers and missing elements simultaneously. In Section 5 we present an extensive simulation study and Section 6 contains the illustration of our methodology on a real life example with scattering. Section 7 summarized our results.

Throughout the paper, we use the same notation as in Smilde et al. [2004] and Engelen and Hubert [2011]:

- arrays and matrices are written in bold capitals and vectors in bold lower case

- the number of samples is denoted by $I$

- for each sample a $J \times K$ matrix $\mathbf{X}_{i}$ is available, which corresponds to the measurements of a set of $J$ and another set of $K$ variables 
- by stacking these matrices we obtain the three-way data array $\underline{\mathbf{X}}$

- when $\underline{\mathbf{X}}$ is unfolded to a matrix, we write $\mathbf{X}$ together with superscripts to identify how the unfolding is performed, e.g. $\mathbf{X}^{(I \times J K)}$

- vec-operator vec $(\mathbf{X})$ is used to represent the vector obtained by unfolding a matrix $\mathbf{X}=\left[\boldsymbol{x}_{1}, \boldsymbol{x}_{2}, \ldots\right]$ column-wise to one column.

\section{Classical PARAFAC and incomplete data}

\subsection{The ALS algorithm for PARAFAC}

To construct a PARAFAC model for three-way data an $(I \times F)$ score matrix $\boldsymbol{A}$ and $(J \times F)$ - and $(K \times F)$-loading matrices $\boldsymbol{B}$ and $\boldsymbol{C}$ are defined, such that the unfolded matrix $\boldsymbol{X}^{I \times J K}$ can be decomposed as:

$$
\boldsymbol{X}^{I \times J K}=\boldsymbol{A}(\boldsymbol{C} \odot \boldsymbol{B})^{\prime}+\boldsymbol{E}^{I \times J K} .
$$

In here, $F$ is the number of factors to include in the model, $\boldsymbol{E}^{I \times J K}$ is the unfolded error term and $\boldsymbol{C} \odot \boldsymbol{B}$ is the column-wise Kathri-Rao product defined as $\boldsymbol{C} \odot \boldsymbol{B}=$ $\left[\operatorname{vec}\left(\boldsymbol{b}_{1} \boldsymbol{c}_{1}^{\prime}\right), \ldots, \operatorname{vec}\left(\boldsymbol{b}_{F} \boldsymbol{c}_{F}^{\prime}\right)\right]$

An estimate of the $i$ th observation for the $j$ th variable and the $k$ th occasion is given by:

$$
\hat{x}_{i j k}=\sum_{f=1}^{F} \hat{a}_{i f} \hat{b}_{j f} \hat{c}_{k f},
$$

which is equivalent to the matrix notation $\hat{\boldsymbol{X}}=\hat{\boldsymbol{A}}(\hat{\boldsymbol{C}} \odot \hat{\boldsymbol{B}})^{\prime}$. Using $\hat{\boldsymbol{X}}$, residuals can be defined as the difference between the estimated and the observed data. The 
residual for observation $i$ is thus given by $\boldsymbol{R}_{i}=\boldsymbol{X}_{i}-\hat{\boldsymbol{X}}_{i}$. The Frobenius norm of $\boldsymbol{R}_{i}$ is called the residual distance $(\mathrm{RD})$ and equals:

$$
R D_{i}=\left\|\boldsymbol{X}_{i}-\hat{\boldsymbol{X}}_{i}\right\|_{F}=\sqrt{\sum_{j=1}^{J} \sum_{k=1}^{K}\left(x_{i j k}-\hat{x}_{i j k}\right)^{2}} .
$$

The scores and loadings of the PARAFAC model are estimated by minimizing the objective function, where $\mathbf{X}=\mathbf{X}^{(I \times J K)}$ unless noted otherwise:

$$
\begin{aligned}
\|\boldsymbol{X}-\hat{\boldsymbol{X}}\|_{F}^{2} & =\left\|\boldsymbol{X}-\hat{\boldsymbol{A}}(\hat{\boldsymbol{C}} \odot \hat{\boldsymbol{B}})^{\prime}\right\|_{F}^{2} \\
& =\sum_{i=1}^{I} \sum_{j=1}^{J} \sum_{k=1}^{K}\left(x_{i j k}-\hat{x}_{i j k}\right)^{2},
\end{aligned}
$$

which is equivalent with minimizing the sum of the squared residual distances. An algorithm based on alternating Least Squares (PARAFAC-ALS) is frequently used for this purpose (see e.g. Smilde et al. [2004]). This means that given initial estimates for $\boldsymbol{B}$ and $\boldsymbol{C}, \boldsymbol{A}$ is estimated conditionally on $\boldsymbol{B}$ and $\boldsymbol{C}$ by minimizing (4). If we define $\boldsymbol{Z}=(\boldsymbol{C} \odot \boldsymbol{B})$ the optimization problem can be reduced to minimizing $\left\|\boldsymbol{X}-\boldsymbol{A} \boldsymbol{Z}^{\prime}\right\|_{F}^{2}$, which gives rise to the classical least squares regression problem. A least squares estimate for $\boldsymbol{A}$ is therefore given by $\hat{\boldsymbol{A}}=\boldsymbol{X} \boldsymbol{Z}\left(\boldsymbol{Z}^{\prime} \boldsymbol{Z}\right)^{+}$with $\left(\boldsymbol{Z}^{\prime} \boldsymbol{Z}\right)^{+}$the Moore-Penrose inverse of $\left(\boldsymbol{Z}^{\prime} \boldsymbol{Z}\right)$. Estimates for $\boldsymbol{B}$ and $\boldsymbol{C}$ are found analogously.

\subsection{Dealing with missing elements}

Missing data frequently occur in a large number of applications in the field of chemometrics ranging from calibration problems to statistical process control. The ALS algorithm can not deal with missing elements and hence some modifications are 
needed. A good method to deal with data containing incomplete observations is the EM algorithm of Dempster et al. [1977].

For analyzing multi-way data sets with missing elements, Bro [1997] and Tomasi and Bro [2004] have presented the following ALS-SI algorithm, which is also based on the EM algorithm and stands for Alternating Least Squares with Single Imputation:

1. Obtain a first estimate $\tilde{\mathbf{X}}^{(0)}$ for $\underline{\mathbf{X}}$ where the missing elements are filled in with the initial estimate (by default the mean of all non-missing values in the whole array is taken).

2. Set $\ell=1$

3. Perform PARAFAC-ALS on $\tilde{\mathbf{X}}^{(\ell-1)}$ (note that apart from the initial estimate $\tilde{\mathbf{X}}^{(0)}, \tilde{\mathbf{X}}$ is different for different number of factors $F$ ), obtain the three loading matrices and obtain a fit $\hat{\mathbf{X}}^{(\ell)}$ by using equation (2).

4. Construct a new estimate $\tilde{\mathbf{X}}^{(\ell)}$ which contains the elements of $\underline{\mathbf{X}}$ which were not missing and the elements of $\hat{\mathbf{X}}^{(\ell)}$ which correspond to the missing elements in $\underline{\mathbf{X}}$.

5. Compare estimates of missing elements from $\tilde{\mathbf{X}}^{(\ell)}$ with the corresponding (former) estimates from $\tilde{\mathbf{X}}^{(\ell-1)}$.

6. If convergence is not attained, increase $\ell$ by 1 and return to step 3 .

It is shown that by using this algorithm a PARAFAC model can still be correctly determined when a large fraction of the data is missing [Tomasi and Bro, 2004]. 


\section{The robust PARAFAC method}

In Engelen and Hubert [2011] it is shown that the least squares estimates in the ALS algorithm are heavily attracted by outliers and a robust PARAFAC version is constructed to prevent this corruption. The robustification of the PARAFAC method consists of three major steps:

1. Look for the $h$ samples (with $\frac{I}{2}<h<I$ ) that minimize the objective function (4).

2. Apply PARAFAC-ALS on these $h$ points.

3. Include a reweighting step to increase the efficiency.

Note that the initial $h$-subset is constructed by performing ROBPCA [Hubert et al.,

2005], a robust PCA method, on the unfolded data matrix $\mathbf{X}^{I \times J K}$. The value of $h$ determines the fraction of outliers the method can resist. The greater this value, the more robust, but also the less statistical efficient the method is. The default value of $h$ is put equal to $75 \%$ of the total number of samples, but can be adapted by the user. The number of components to retain in ROBPCA are chosen by looking at the screeplot and the robust PRESS [Hubert and Engelen, 2007].

Note that it is possible to divide all the samples into four classes: regular samples, residual outliers, good leverage points and bad leverage points. The classification of the observations is characterized by two distances, namely the residual distance (RD) and the score distance (SD). The RD has been introduced in (3) and can be 
considered as a measure of how well the fitted data and the observed data collapse, whereas the SD measures how much the estimated scores of a sample deviate from the center of the scores. The SD of an observation $\boldsymbol{X}_{i}$ is computed by taking the robust counterpart of the Mahalanobis distance of the score $\hat{\boldsymbol{a}}_{i}$ to the center of the scores matrix $\hat{\boldsymbol{A}}$ :

$$
\mathrm{SD}_{i}=\sqrt{\left(\hat{\boldsymbol{a}}_{i}-\hat{\boldsymbol{\mu}}\right)^{\prime} \hat{\boldsymbol{\Sigma}}^{-1}\left(\hat{\boldsymbol{a}}_{i}-\hat{\boldsymbol{\mu}}\right)}
$$

with $\hat{\boldsymbol{\mu}}$ and $\hat{\boldsymbol{\Sigma}}$ robust estimates of the center and covariance matrix of the scores respectively, which are provided by the Minimum Covariance Determinant (MCD) method [Rousseeuw, 1984]. Observations are considered as outliers if their SD or RD is too large. Appropriate cutoff values are provided in [Engelen and Hubert, 2011]. Note that these outlier detection rules do not depend on the order or the sign of the loadings, but only on the subspace spanned by the loadings.

A regular point is a sample for which the RD as well as the SD are smaller than the corresponding cutoff value. Observations which have a highly deviating underlying structure, but a small SD are called residual outliers. The leverage points have a large $\mathrm{SD}$, but the RD is small for good leverage points, whereas a large RD typifies the bad leverage points. To visualize this classification, an outlier map can be made, where the $\mathrm{RD}$ is plotted against the $\mathrm{SD}$, together with a vertical and horizontal line for the cutoff values. This will be illustrated in Section 6 on the Dorrit data set. 


\section{Robust PARAFAC for incomplete data}

As mentioned before, Serneels and Verdonck [2008] have already applied the EM algorithm to obtain a robust PCA method (ER-ROBPCA) that can cope with missing data. Note that in the robust case the estimates are obtained by means of a robust estimation technique (instead of via maximum likelihood) in the maximization step and therefore this step is then often denoted as R-step (of the ER algorithm) [Little, 1988]. A similar strategy can be followed to construct a robust PARAFAC method that can cope with outlying cases and missing elements simultaneously. This procedure yields the following algorithm, denoted as RPARAFAC-SI since it is also based on starting with a single imputation:

1. An initial $h$-subset is constructed by performing ER-ROBPCA on the unfolded data matrix $\mathbf{X}^{(I \times J K)}$. As ER-ROBPCA needs an initial imputation value, we take the average of the medians of the matching row and column. The $h$ observations with the smallest residual distance towards the space spanned by the robustly estimated principal components, are taken as initial guess for the final $h$-subset, that should minimize (4).

2. The ER-ROBPCA step also yields an estimate $\hat{\mathbf{X}}^{(I \times J K)}$ for the unfolded data matrix $\mathbf{X}^{(I \times J K)}$. Use this to obtain a fit $\tilde{\mathbf{X}}^{(0)}$ for $\underline{\mathbf{X}}$ which contains the elements of $\underline{\mathbf{X}}$ which were not missing and the elements of $\hat{\mathbf{X}}^{(0)}$ which correspond to the missing elements in $\underline{\mathbf{X}}$.

3. Set $\ell=1$. 
4. Perform PARAFAC-ALS on the current $h$ observations of $\tilde{\mathbf{X}}^{(\ell-1)}$, obtain the three loading matrices and obtain a fit $\hat{\mathbf{X}}^{(\ell)}$ by using equation (2) for all samples $i=1, \ldots, n$.

5. Construct a new estimate $\tilde{\mathbf{X}}^{(\ell)}$ which contains the elements of $\underline{\mathbf{X}}$ which were not missing and the elements of $\hat{\mathbf{X}}^{(\ell)}$ which correspond to the missing elements in $\underline{\mathbf{X}}$.

6. Compute the residual distances for each observation $i=1, \ldots, n$ :

$$
R D_{i}=\left\|\tilde{\mathbf{X}}_{i}^{(\ell)}-\hat{\mathbf{X}}_{i}^{(\ell)}\right\|_{F}
$$

Create a new $h$-subset by storing the $h$ samples with smallest residual distance.

7. Compare estimates of missing elements from $\tilde{\mathbf{X}}^{(\ell)}$ with the corresponding (former) estimates from $\tilde{\mathbf{X}}^{(\ell-1)}$. If convergence is not attained, increase $\ell$ by 1 and return to step 4.

8. If convergence is attained, a reweighting step is performed: apply PARAFACALS on all the observations (where the missing elements are filled in via the PARAFAC model) with residual distance smaller than the appropriate cutoff.

Note that in step 2 of this algorithm the initial estimates for the missing elements are obtained from ER-ROBPCA, whereas in the next iteration steps the missing values are filled in via the PARAFAC model.

Each iteration (step 4-7) decreases the objective function, so the algorithm will always converge to a (possibly local) optimum. This can be seen as follows: the aim 
of the procedure is to minimize the objective function

$$
\sum_{i \in H} \sum_{j=1}^{J} \sum_{k=1}^{K}\left(\tilde{x}_{i j k}-\hat{x}_{i j k}\right)^{2}
$$

over all $h$-subsets $H$, and all matrices $A, B$ and $C$. Here, $\hat{x}_{i j k}=\sum_{f=1}^{F} \hat{a}_{i f} \hat{b}_{j f} \hat{c}_{k f}$ is the estimated value for entry $x_{i j k}$ whereas $\tilde{x}_{i j k}=\hat{x}_{i j k}$ if entry $x_{i j k}$ is missing and $\tilde{x}_{i j k}=x_{i j k}$ otherwise. Denote

$$
E_{3}^{(\ell-1)}=\sum_{i \in H^{(\ell-1)}} \sum_{j=1}^{J} \sum_{k=1}^{K}\left(\tilde{x}_{i j k}^{(\ell-1)}-\hat{x}_{i j k}^{(\ell-1)}\right)^{2}
$$

the value of the objective function after the $(\ell-1)$-th iteration, where $H^{(\ell-1)}, \tilde{x}_{i j k}^{(\ell-1)}$, and $\hat{x}_{i j k}^{(\ell-1)}$ are the values of $H, \tilde{x}_{i j k}$ and $\hat{x}_{i j k}$ at that point.

1. In step 4 of the algorithm, we find matrices $\hat{A}^{(\ell)}, \hat{B}^{(\ell)}$, and $\hat{C}^{(\ell)}$ such that

$$
E_{1}^{(\ell)}=\sum_{i \in H^{(\ell-1)}} \sum_{j=1}^{J} \sum_{k=1}^{K}\left(\tilde{x}_{i j k}^{(\ell-1)}-\hat{x}_{i j k}^{(\ell)}\right)^{2}
$$

is minimized, with $\hat{x}_{i j k}^{(l)}=\sum_{f=1}^{F} \hat{a}_{i f}^{(l)} \hat{b}_{j f}^{(l)} \hat{c}_{k f}^{(l)}$. Obviously, $E_{1}^{(\ell)} \leqslant E_{3}^{(\ell-1)}$ must hold.

2. In step 5 of the algorithm we obtain new values $\tilde{x}_{i j k}^{(\ell)}$. If entry $x_{i j k}$ is not missing from the data,

$$
\tilde{x}_{i j k}^{(\ell)}-\hat{x}_{i j k}^{(\ell)}=\tilde{x}_{i j k}^{(\ell-1)}-\hat{x}_{i j k}^{(\ell)}
$$

will hold and that entry will not cause the objective function to change. If $x_{i j k}$ is missing however, we set $\tilde{x}_{i j k}^{(\ell)}=\hat{x}_{i j k}^{(\ell)}$, causing a decrease in the objective function. As such,

$$
E_{2}^{(\ell)}=\sum_{i \in H^{(\ell-1)}} \sum_{j=1}^{J} \sum_{k=1}^{K}\left(\tilde{x}_{i j k}^{(\ell)}-\hat{x}_{i j k}^{(\ell)}\right)^{2} \leqslant E_{1}^{(\ell)} .
$$


3. Finally, in step 6 we choose the new subset $H^{(\ell)}$ consisting of the observations with the smallest residual distances

$$
\sum_{j=1}^{J} \sum_{k=1}^{K}\left(\tilde{x}_{i j k}^{(\ell)}-\hat{x}_{i j k}^{(\ell)}\right)^{2}
$$

so that

$$
E_{3}^{(\ell)}=\sum_{i \in H^{(\ell)}} \sum_{j=1}^{J} \sum_{k=1}^{K}\left(\tilde{x}_{i j k}^{(\ell)}-\hat{x}_{i j k}^{(\ell)}\right)^{2} \leqslant E_{2}^{(\ell)},
$$

showing that in each iteration of the algorithm the objective function decreases.

We have also tested the following alternative approaches, but their performance was significantly worse than our final algorithm:

Method 2 In step 4, PARAFAC-ALS is performed on the current $h$ points of $\tilde{\mathbf{X}}^{(0)}$ instead of $\tilde{\mathbf{X}}^{(\ell-1)}$. The reweighting step is also performed on all the observations of $\tilde{\mathbf{X}}^{(0)}$ with small residual distance. This method has a worse performance than the final algorithm because it uses the values obtained by the ER-ROBPCA step as imputations for the missing values during the rest of the algorithm without updating them in each PARAFAC-ALS step to reflect the new 'best' estimates.

Method 3 The initial ER-ROBPCA step is used only to obtain an initial $h$ subset, no estimates of the missing values are performed. In step 4, PARAFAC that can cope with missing elements is performed on the current $h$ points of $\underline{\mathbf{X}}$ (in particular we used ALS-SI) instead of PARAFAC-ALS on the current $h$ points of $\tilde{\mathbf{X}}^{(\ell-1)}$. The reweighting step is also performed on all the observations of $\underline{\mathbf{X}}$ 
with small residual distance. Although this method has a performance which is comparable to the final algorithm, we have observed that the computational time for this alternative approach is worse. Moreover, we have observed that the algorithm failed to converge for a few simulation runs, resulting in poor results and a 100-fold increase in computation time. An analysis of the specific datasets, where this behavior occurred, revealed that this is caused by a concentration of missing entries in some of the nodes.

\section{Simulation study}

In this section we investigate the behavior of the robust PARAFAC algorithm that can deal with missing elements in a simulation experiment.

\subsection{General setup}

The simulaton design is based on the setup of Engelen and Hubert [2011], but we also add missing elements in the data cube. We first generate three-way data with a given number of factors $F$ following the PARAFAC model (1) where the true scores $\boldsymbol{A}$ and loadings $\boldsymbol{B}$, and $\boldsymbol{C}$ are drawn from a multivariate random normal distribution $N_{F}\left(\mathbf{0}, \boldsymbol{\Sigma}_{F}\right)$ with $\boldsymbol{\Sigma}_{F}$ a diagonal matrix with $\left(\begin{array}{ll}10 & 2\end{array}\right)$ as diagonal elements. $\boldsymbol{B}$ and $\boldsymbol{C}$ are rescaled such that their Frobenius norm is equal to one (this can be done without loss of generality, because of the scale indeterminacy of the PARAFAC model). The 
added noise term $\boldsymbol{E}^{I \times J K}$ is defined as [Tomasi and Bro, 2006]

$$
\boldsymbol{E}^{I \times J K}=\frac{\text { Noise } \%}{100-\text { Noise } \%}\left\|\boldsymbol{X}_{\text {pure }}^{I \times J K}\right\|_{F} \tilde{\boldsymbol{E}}^{I \times J K}
$$

where $\tilde{\boldsymbol{E}}^{I \times J K}$ is a matrix of standard normally distributed random variables having a Frobenius norm of 1 . Noise $\%$ defines the percentage of noise in the total variance of the data $\boldsymbol{X}$ and is always set to $20 \%$. We generate uncontaminated and contaminated data. In order to contaminate the data, we replace $100 \varepsilon \%$ of the observations by different types of outliers. The contaminated part of the data is constructed as follows:

- Residual outliers: adding a constant $c_{1}=0.1$ to $100 \varepsilon \%$ of $\boldsymbol{X}$.

- Good leverage points: multiplying $100 \varepsilon \%$ of $\boldsymbol{A}(\boldsymbol{C} \odot \boldsymbol{B})^{\prime}$ with a constant $c_{2}=10$ before adding the noise term.

- Bad leverage points: adding $c_{3}=0.2$ to the good leverage points.

Missing values were then added by randomly replacing the desired fraction of elements of $\boldsymbol{X}$ by NaN's, so that in the simulation the MAR assumption on missingness holds. Alternatively we also consider a second pattern where various entire tubes are simultaneously set as missing (the selection of the tubes is done at random). This means that, for specific combinations of $j$ and $k$, we set $x_{i j k}$ as missing for each $i=1, \ldots, I$. This situation can arise if, in excitation-emission measurements, the instrument fails to make a measurement at specific combinations of excitation and emission frequencies. It can also occur when scatter has been identified in all samples and is set to missing values. This will be illustrated in Section 6 . 
In order to evaluate the proposed methodology, the following criteria were used:

(A) The mean squared error of the regular observed measurements:

$$
M S E=\frac{1}{w J K} \sum_{i=1}^{I} \sum_{j=1}^{J} \sum_{k=1}^{K} w_{i}\left(x_{i j k}-\hat{x}_{i j k}\right)^{2}
$$

where $w_{i}$ indicates whether sample $i$ is a regular point $\left(w_{i}=1\right)$ or an outlier $\left(w_{i}=0\right)$ and $w=\sum_{i=1}^{I} w_{i}$. If the element $x_{i j k}$ is missing, it is not added in the MSE.

(B) The product of the congruences (i.e. the cosine of the angle between the true and estimated loadings) for the single loading matrix $B$. Note that we had to consider all possible permutations of the estimated loadings, because their order is not uniquely defined. For finding the optimal permutation we followed the approach of [Tomasi and Bro, 2004].

(C) The product of the congruences for the loading matrix $C$.

For each situation, 100 data sets are generated and $I=100, J=100, K=10$ and $F=2$ are chosen as data dimensions and complexity. We consider percentages of contamination $\varepsilon=0,10 \%$ and $20 \%$ for data containing $0 \%, 10 \%$ and $20 \%$ of missings. In all simulations the parameter $h$, denoting the fraction of outliers the robust methods can resist, is set equal to the default value of $h=\lfloor 0.75 I\rfloor=75$. 


\subsection{Simulation results}

Means over 50 simulations and their respective standard errors (between brackets) of each of these simulation settings are reported in a set of tables. In each table, results for the robust RPARAFAC-SI and the classical ALS-SI are presented with respect to the different types and varying percentages of outliers. Table I considers the situation without missing elements, Tables II and III list the results for $10 \%$ and $20 \%$ missing elements, placed at random, whereas Tables IV and V show the behaviour of the methods at the missing tube setting.

Overall, it can be observed from the first line of each table that ALS-SI and RPARAFAC yield approximately the same results when the data are not contaminated. Hence, applying the robust PARAFAC method that can cope with missings to uncontaminated incomplete data seems to be safe.

When adding good leverage points to the data, we see that both methods give comparable results according to the MSE criterion (A) and that classical ALSSI often obtains better estimates for the $B$ and $C$ loadings. Since good leverage points still follow the model, including them in the estimation procedure increases the precision of the estimates (explaining the good results of classical PARAFAC). However, robust PARAFAC succeeds in detecting the good leverage points and then yields estimates that are comparable with those of uncontaminated data (after excluding the outliers from the model).

As soon as residual outliers or bad leverage points are included in the data, ALS-SI yields higher MSE values and much lower congruences for the $B$ and $C$ - 
loadings, whereas RPARAFAC-SI still yields reliable results. It is clear that similar to PARAFAC-ALS, also ALS-SI is influenced by any type and amount of contamination.

Summarized we see that the good results for robust PARAFAC carry through to incomplete data if we apply our proposed methodology.

[Table I - V about here]

\section{Dorrit data}

In this section we apply our new method for incomplete data to the Dorrit dataset, as considered in Engelen et al. [2009]. The data set contains $I=27$ mixtures of four known fluorophores: phenylanaline, 3, 4-dihydroxyphenylalanine (DOPA), 1, 4-dihydroxybenzene and tryptophan. For every sample an excitation-emission matrix was obtained by measuring the emission spectra from 250 to $482 \mathrm{~nm}$ at $2 \mathrm{~nm}$ intervals (yielding $K=116$ ), with excitation at every $5 \mathrm{~nm}$ from 230 to $315 \mathrm{~nm}$ on a Perkin-Elmer LS50 B fluorescence spectrometer (hence $J=18$ ). From previous investigations, it is know that $F=4$ components are appropriate and that some EEM landscapes can be considered as outliers. Moreover this data set contains scatter, which affects all samples, as well as several missing values. In Engelen et al. [2009] a procedure is developed which can deal with both outlying samples and scatter, but not yet with missing values. Here we improve their method by including our new algorithm. 


\subsection{Methodology}

To analyse these data, we first use an automatic procedure to detect the scatter, as described in Engelen et al. [2007], and set the detected elements to missing values. Note that this automatic scatter detection method relies on applying ROBPCA on all the data slices $\underline{\boldsymbol{X}}(:, j,:)$ and $\underline{\boldsymbol{X}}(:,:, k)$ for $j=1, \ldots, J$ and $k=1, \ldots, K$. As there are missing elements in many slices, we could always use ER-ROBPCA for incomplete data, but this is very time consuming. Hence, we first perform RPARAFAC-SI on the original data and use the imputed values to perform ROBPCA for complete data on each slice. The result of this analysis is a set of cells from the cube which are flagged as scatter and which are set to missing.

After this step, we perform a trilinear decomposition using the PARAFAC algorithm. If we would apply the classical ALS-SI after removing the scatter, the final results would still be corrupted due to the presence of the obviously outlying observations 2, 3 and 5. Instead of removing these a priori, we use RPARAFAC-SI to deal with these outlying samples, as well as the missing entries, in an appropriate way.

We compare the results of our proposed procedure with the procedure used in Engelen et al. [2009]. The main differences between the two procedures are:

- In Engelen et al. [2009], the elements indicating scatter are first also set to missing, but then the data are interpolated in all missing values. Moreover we do not need to perform an imputation of the missing values in the original 
data.

- The procedure used in Engelen et al. [2009] repeats the scatter detection and robust PARAFAC steps a few times, where in each step, the scatter detection is performed using only those observations flagged as regular observations in the preceding robust PARAFAC step.

\subsection{Results}

We first compare the mean squared errors of the models obtained with both methods. These MSEs are computed based on the non-missing, non-scatter elements of the data array, with the observations flagged as outliers excluded (for the two robust PARAFAC methods). The method described in Engelen et al. [2009] achieves an MSE of 89.81, while our newly proposed method achieves an MSE of 16.79, a 81.3\% reduction due to treating the scatter as missing elements instead of imputing values for them.

Secondly, we can look at the outlier map in Figure 1: our method identifies observations 2, 3, 4, 5 as bad leverage points, 10 as a residual outlier and cases 12 and 16 as good leverage points. These observations, except observation 4, are known to be unambiguous outliers from previous investigations. Observation 10 is not identified as a leverage point which is consistent with the analysis in Engelen and Hubert [2011] and Engelen et al. [2009]. Although observation 4 has been flagged as a bad leverage point, it is more or less a border case as well. Nevertheless, it seems that flagging this observation as an outlier, and treating the scatter as missing elements 
results in a significant decrease in MSE.

[Figure 1 about here]

Next, we consider the loadings. Due to the way the Dorrit dataset is obtained, we would expect to see four distinct spectra in both the $B$ and $C$ loadings. The results can be seen in the top row of Figure 2. Clearly, our method also manages to correctly identify the four different spectra. If we compute the congruence coefficients for the estimated emission and excitation loadings and the real ones (see Engelen and Hubert [2011], Figure 13), we find values of 0.9932 and 0.9999 respectively, signifying a nearly perfect match between the estimated loadings and the actual spectra of the compounds in the mixtures. This is better than the congruences obtained with the method from Engelen et al. [2009]. The loadings from this approach are depicted in the lower row of Figure 2, and yield congruences of 0.9812 and 0.9794 for the emission and excitation loadings.

[Figure 2 about here]

Finally, we check whether or not the estimated scores (the A matrix) correspond to the quantities of the compounds in the mixtures. We can do this by making a scatterplot of the estimated scores versus the actual quantities of the compounds, for each of the four compounds. If the points lie more or less on a line, it means that the estimated scores correspond well to the actual mixture quantities of the components. These plots can be seen in Figure 3. 
[Figure 3 about here]

We observe that the regular observations, the dots in the plots, lie quite well on a line, so the estimated scores are proportional to the real quantities of the compounds in the mixture. If we compute the correlation between the real quantities and the estimated scores, we find correlations of $0.9974,0.9939,0.9933$, and 0.9893 for each respective component, if we restrict ourselves to the non-outlying observations, once again indicating that we are able to find the real mixture quantities.

We have also repeated our analysis with $F=6$ components instead of 4 . If we do this, we observe that the first four components are very similar to the ones we have found in the previous analysis, and that the fifth and sixth components correspond to spikes in the emission spectra, at different frequencies, leading us to believe that part of the scattering is modelled now. Also, if we compute the mean squared error for this PARAFAC model, we see that it decreases the MSE further to 11.49, and that the congruence coefficients of the emission and excitation loadings, compared to the true loadings, stay almost constant at 0.9926 and 0.9999 . This indicates that selecting more components than needed does not influence the accuracy of the estimates.

\section{Conclusion}

In this paper we have developed a robust PARAFAC method which can deal with outlying samples and with missing elements. It is based on the EM-methodology and 
the robust PARAFAC method for complete data from Engelen and Hubert [2011]. Simulations showed that the method remains robust and adequate when contamination is present in incomplete data. In particular we applied our new method on a fluorescence data set which contains scatter and outlying EEM landscapes. If we first automatically detect the scatter and set the flagged scatter to missing values, we are able to well estimate the underlying PARAFAC model and to find the abnormal samples at the same time.

\section{References}

R. Bro. PARAFAC. Tutorial and applications. Chemometrics and Intelligent Laboratory Systems, 38:149-171, 1997.

T.-C. Cheng and M. Victoria-Feser. High breakdown estimation of multivariate location and scale with missing observations. British Journal of Mathematical and Statistical Psychology, 55:317-335, 2002.

A.P. Dempster, N.M. Laird, and D.B. Rubin. Maximum likelihood for incomplete data via the em algorithm (with discussions). Journal of the Royal Statistical Society B, 39:1-38, 1977.

S. Engelen and M. Hubert. Detecting outlying samples in a parallel factor analysis model. Analytica Chemica Acta, 705:155-165, 2011.

S. Engelen, S. Frosch Møller, and M. Hubert. Automatically identifying scatter in 
fluorescence data using robust techniques. Chemometrics and Intelligent Laboratory Systems, 86:35-51, 2007.

S. Engelen, S. Frosch Møller, and B.M. Jorgensen. A fully robust PARAFAC method for analyzing fluorescence data. Journal of Chemometrics, 23:124-131, 2009.

M. Hubert and S. Engelen. Fast cross-validation for high-breakdown resampling algorithms for PCA. Computational Statistics and Data Analysis, 51:5013-5024, 2007.

M. Hubert, P.J. Rousseeuw, and K. Vanden Branden. ROBPCA: a new approach to robust principal components analysis. Technometrics, 47:64-79, 2005.

M. Hubert, P.J. Rousseeuw, and S. Van Aelst. High breakdown robust multivariate methods. Statistical Science, 23:92-119, 2008.

P.M. Kroonenberg. Applied multi-way data analysis. Wiley-Interscience, Hoboken,NJ, 2008.

R.J.A. Little. Robust estimation of the mean and covariance matrix from data with missing values. Applied Statistics, 37:23-38, 1988.

R.J.A. Little and D.B. Rubin. Statistical Analysis with Missing Data. WileyInterscience, New York, 1987.

P.J. Rousseeuw. Least median of squares regression. Journal of the American Statistical Association, 79:871-880, 1984. 
S. Serneels and T. Verdonck. Principal component analysis for data containing outliers and missing elements. Computational Statistics and Data Analysis, 52: $1712-1727,2008$.

S. Serneels and T. Verdonck. Principal component regression for data containing outliers and missing elements. Computational Statistics and Data Analysis, 53: 3855-3863, 2009.

A. Smilde, R. Bro, and P. Geladi. Multi-way Analysis with Applications in the Chemical Sciences. Wiley, England, 2004.

I. Stanimirova, S. Serneels, P. Van Espen, and B. Walczak. How to construct a multiple regression model for data with missing elements and outlying objects. Analytica Chimica Acta, 581(2):324-332, 2007.

G. Tomasi and R. Bro. PARAFAC and missing values. Chemometrics and Intelligent Laboratory Systems, 75:163-180, 2004.

G. Tomasi and R. Bro. A comparison of algorithms for fitting the PARAFAC model. Computational Statistics and Data analysis, 50:1700-1734, 2006.

B. Walczak and D.L. Massart. Tutorial, dealing with missing data, part I. Chemometrics and Intelligent Laboratory Systems, 58:15-27, 2001. 


\section{List of Tables}

Table I: Simulation results for data with $0 \%$ of missing elements.

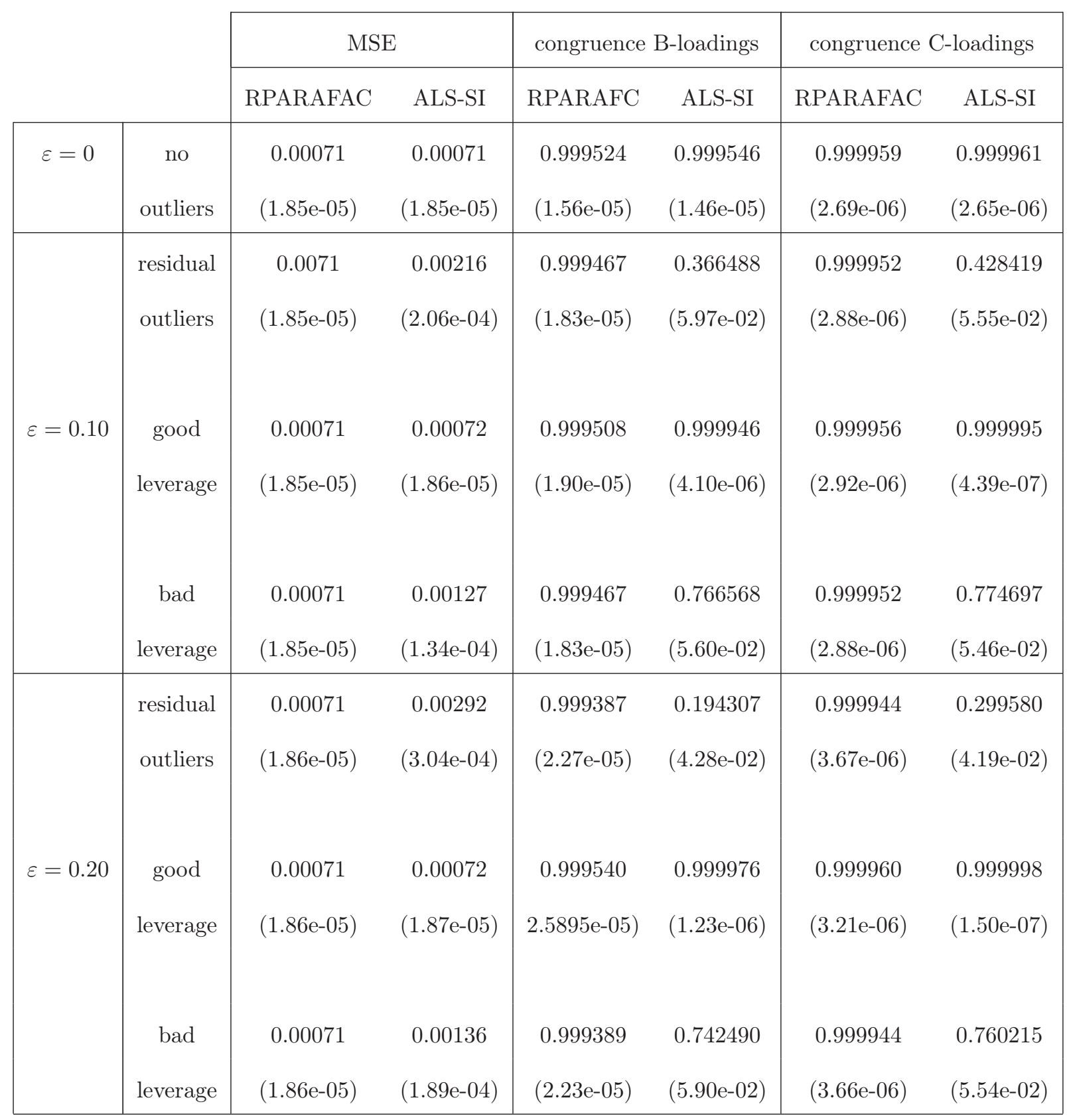


Table II: Simulation results for data with $10 \%$ of missing elements.

\begin{tabular}{|c|c|c|c|c|c|c|c|}
\hline & \multicolumn{2}{|c|}{ MSE } & \multicolumn{2}{|c|}{ congruence B-loadings } & \multicolumn{2}{|c|}{ congruence C-loadings } \\
\hline & & RPARAFAC & ALS-SI & RPARAFAC & ALS-SI & RPARAFAC & ALS-SI \\
\hline$\varepsilon=0$ & $\begin{array}{c}\text { no } \\
\text { outliers }\end{array}$ & $\begin{array}{c}0.00071 \\
(1.85 \mathrm{e}-05)\end{array}$ & $\begin{array}{c}0.00071 \\
(1.85 \mathrm{e}-05)\end{array}$ & $\begin{array}{c}0.999460 \\
(1.75 \mathrm{e}-05)\end{array}$ & $\begin{array}{c}0.999494 \\
(1.60 \mathrm{e}-05)\end{array}$ & $\begin{array}{c}0.999956 \\
(2.70 \mathrm{e}-06)\end{array}$ & $\begin{array}{r}0.999959 \\
(2.59 \mathrm{e}-06)\end{array}$ \\
\hline \multirow{3}{*}{$\varepsilon=0.10$} & $\begin{array}{l}\text { residual } \\
\text { outliers }\end{array}$ & $\begin{array}{c}0.00071 \\
(1.86 \mathrm{e}-05)\end{array}$ & $\begin{array}{c}0.00235 \\
(1.87 \mathrm{e}-04)\end{array}$ & $\begin{array}{c}0.999401 \\
(2.06 \mathrm{e}-05)\end{array}$ & $\begin{array}{c}0.275924 \\
(5.24 \mathrm{e}-02)\end{array}$ & $\begin{array}{c}0.999949 \\
(2.88 \mathrm{e}-06)\end{array}$ & $\begin{array}{r}0.337312 \\
(4.96 \mathrm{e}-02)\end{array}$ \\
\hline & $\begin{array}{c}\text { good } \\
\text { leverage }\end{array}$ & $\begin{array}{c}0.00071 \\
(1.86 \mathrm{e}-05)\end{array}$ & $\begin{array}{c}0.00071 \\
(1.85 \mathrm{e}-05)\end{array}$ & $\begin{array}{c}0.999464 \\
(2.24 \mathrm{e}-05)\end{array}$ & $\begin{array}{c}0.981561 \\
(1.84 \mathrm{e}-02)\end{array}$ & $\begin{array}{c}0.999955 \\
(2.84 \mathrm{e}-06)\end{array}$ & $\begin{array}{r}0.980490 \\
(1.95 \mathrm{e}-02)\end{array}$ \\
\hline & $\begin{array}{c}\text { bad } \\
\text { leverage }\end{array}$ & $\begin{array}{c}0.00071 \\
(1.86 \mathrm{e}-05)\end{array}$ & $\begin{array}{c}0.00133 \\
(1.57 \mathrm{e}-04)\end{array}$ & $\begin{array}{c}0.999401 \\
(2.08 \mathrm{e}-05)\end{array}$ & $\begin{array}{c}0.762263 \\
(5.69 \mathrm{e}-02)\end{array}$ & $\begin{array}{c}0.999949 \\
(2.85 \mathrm{e}-06)\end{array}$ & $\begin{array}{c}0.777701 \\
(5.38 \mathrm{e}-02)\end{array}$ \\
\hline \multirow{3}{*}{$\varepsilon=0.20$} & $\begin{array}{l}\text { residual } \\
\text { outliers }\end{array}$ & $\begin{array}{c}0.00071 \\
(1.86 \mathrm{e}-05)\end{array}$ & $\begin{array}{c}0.00299 \\
(2.47 \mathrm{e}-04)\end{array}$ & $\begin{array}{c}0.999316 \\
(2.50 \mathrm{e}-05)\end{array}$ & $\begin{array}{c}0.147651 \\
(3.15 \mathrm{e}-02)\end{array}$ & $\begin{array}{c}0.999940 \\
(3.62 \mathrm{e}-06)\end{array}$ & $\begin{array}{r}0.247325 \\
(3.38 \mathrm{e}-02)\end{array}$ \\
\hline & $\begin{array}{c}\text { good } \\
\text { leverage }\end{array}$ & $\begin{array}{c}0.00071 \\
(1.86 \mathrm{e}-05)\end{array}$ & $\begin{array}{c}0.00072 \\
(1.87 \mathrm{e}-05)\end{array}$ & $\begin{array}{c}0.999486 \\
(2.88 \mathrm{e}-05)\end{array}$ & $\begin{array}{c}0.999973 \\
(1.40 \mathrm{e}-06)\end{array}$ & $\begin{array}{c}0.999956 \\
(3.57 \mathrm{e}-06)\end{array}$ & $\begin{array}{r}0.999998 \\
(1.78 \mathrm{e}-07)\end{array}$ \\
\hline & $\begin{array}{c}\text { bad } \\
\text { leverage }\end{array}$ & $\begin{array}{c}0.00071 \\
(1.86 \mathrm{e}-05)\end{array}$ & $\begin{array}{c}0.00163 \\
(3.02 \mathrm{e}-04)\end{array}$ & $\begin{array}{c}0.999317 \\
(2.49 \mathrm{e}-05)\end{array}$ & $\begin{array}{c}0.708033 \\
(6.08 \mathrm{e}-02)\end{array}$ & $\begin{array}{c}0.999940 \\
(3.60 \mathrm{e}-06)\end{array}$ & $\begin{array}{c}0.720391 \\
(5.87 \mathrm{e}-02)\end{array}$ \\
\hline
\end{tabular}


Table III: Simulation results for data with $20 \%$ of missing elements.

\begin{tabular}{|c|c|c|c|c|c|c|c|}
\hline & \multicolumn{2}{|c|}{ MSE } & \multicolumn{2}{|c|}{ congruence B-loadings } & \multicolumn{2}{|c|}{ congruence C-loadings } \\
\hline & & RPARAFAC & ALS-SI & RPARAFAC & ALS-SI & RPARAFAC & ALS-SI \\
\hline$\varepsilon=0$ & $\begin{array}{c}\text { no } \\
\text { outliers }\end{array}$ & $\begin{array}{c}0.00071 \\
(1.86 \mathrm{e}-05)\end{array}$ & $\begin{array}{c}0.00071 \\
(1.86 \mathrm{e}-05)\end{array}$ & $\begin{array}{c}0.999386 \\
(1.97 \mathrm{e}-05)\end{array}$ & $\begin{array}{c}0.999434 \\
(1.72 \mathrm{e}-05)\end{array}$ & $\begin{array}{l}0.999949 \\
(3.42 \mathrm{e}-06)\end{array}$ & $\begin{array}{r}0.999953 \\
(2.90 \mathrm{e}-06)\end{array}$ \\
\hline \multirow{3}{*}{$\varepsilon=0.10$} & $\begin{array}{l}\text { residual } \\
\text { outliers }\end{array}$ & $\begin{array}{c}0.00071 \\
(1.86 \mathrm{e}-05)\end{array}$ & $\begin{array}{c}0.00226 \\
(1.91 \mathrm{e}-04)\end{array}$ & $\begin{array}{c}0.999322 \\
(2.38 \mathrm{e}-05)\end{array}$ & $\begin{array}{c}0.319104 \\
(5.52 \mathrm{e}-02)\end{array}$ & $\begin{array}{c}0.999942 \\
(3.60 \mathrm{e}-06)\end{array}$ & $\begin{array}{c}0.373945 \\
(5.25 \mathrm{e}-02)\end{array}$ \\
\hline & $\begin{array}{c}\text { good } \\
\text { leverage }\end{array}$ & $\begin{array}{c}0.00071 \\
(1.86 \mathrm{e}-05)\end{array}$ & $\begin{array}{c}0.00072 \\
(1.83 \mathrm{e}-05)\end{array}$ & $\begin{array}{c}0.999377 \\
(2.27 \mathrm{e}-05)\end{array}$ & $\begin{array}{c}0.989652 \\
(1.03 \mathrm{e}-02)\end{array}$ & $\begin{array}{c}0.999947 \\
(3.43 \mathrm{e}-06)\end{array}$ & $\begin{array}{r}0.989825 \\
(1.02 \mathrm{e}-02)\end{array}$ \\
\hline & $\begin{array}{c}\text { bad } \\
\text { leverage }\end{array}$ & $\begin{array}{c}0.00071 \\
(1.86 \mathrm{e}-05)\end{array}$ & $\begin{array}{c}0.00133 \\
(1.53 \mathrm{e}-04)\end{array}$ & $\begin{array}{c}0.999322 \\
(2.38 \mathrm{e}-05)\end{array}$ & $\begin{array}{c}0.760347 \\
(5.73 \mathrm{e}-02)\end{array}$ & $\begin{array}{c}0.999942 \\
(3.51 \mathrm{e}-06)\end{array}$ & $\begin{array}{c}0.778586 \\
(5.37 \mathrm{e}-02)\end{array}$ \\
\hline \multirow{3}{*}{$\varepsilon=0.20$} & $\begin{array}{l}\text { residual } \\
\text { outliers }\end{array}$ & $\begin{array}{c}0.00071 \\
(1.86 \mathrm{e}-05)\end{array}$ & $\begin{array}{c}0.00299 \\
(2.49 \mathrm{e}-04)\end{array}$ & $\begin{array}{c}0.999239 \\
(2.81 \mathrm{e}-05)\end{array}$ & $\begin{array}{c}0.148892 \\
(3.14 \mathrm{e}-02)\end{array}$ & $\begin{array}{c}0.999933 \\
(4.26 \mathrm{e}-06)\end{array}$ & $\begin{array}{r}0.250588 \\
(3.37 \mathrm{e}-02)\end{array}$ \\
\hline & $\begin{array}{c}\text { good } \\
\text { leverage }\end{array}$ & $\begin{array}{c}0.00071 \\
(1.87 \mathrm{e}-05)\end{array}$ & $\begin{array}{c}0.00072 \\
(1.87 \mathrm{e}-05)\end{array}$ & $\begin{array}{l}0.999425 \\
(3.15 \mathrm{e}-05)\end{array}$ & $\begin{array}{c}0.999969 \\
(1.46 \mathrm{e}-06)\end{array}$ & $\begin{array}{c}0.999949 \\
(4.12 \mathrm{e}-06)\end{array}$ & $\begin{array}{r}0.999997 \\
(2.04 \mathrm{e}-07\end{array}$ \\
\hline & $\begin{array}{c}\text { bad } \\
\text { leverage }\end{array}$ & $\begin{array}{c}0.00071 \\
(1.86 \mathrm{e}-05)\end{array}$ & $\begin{array}{c}0.001381 \\
(1.89 \mathrm{e}-04)\end{array}$ & $\begin{array}{c}0.999241 \\
(2.83 \mathrm{e}-05)\end{array}$ & $\begin{array}{c}0.724735 \\
(5.99 \mathrm{e}-02)\end{array}$ & $\begin{array}{c}0.999933 \\
(4.28 \mathrm{e}-06)\end{array}$ & $\begin{array}{r}0.751098 \\
(5.50 \mathrm{e}-02)\end{array}$ \\
\hline
\end{tabular}


Table IV: Simulation results for data with 10\% of missing tubes.

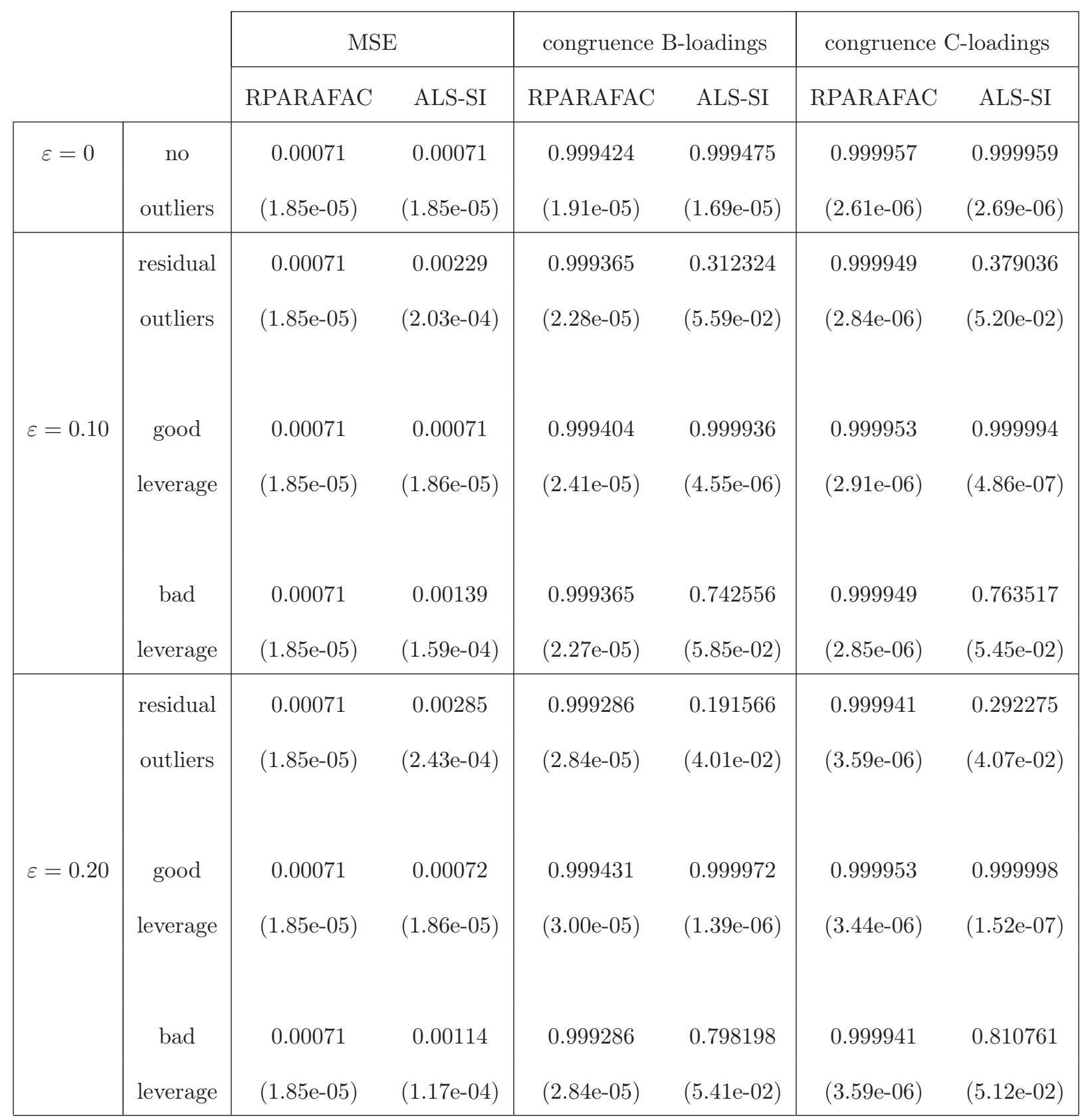


Table V: Simulation results for data with $20 \%$ of missing tubes.

\begin{tabular}{|c|c|c|c|c|c|c|c|}
\hline & \multicolumn{2}{|c|}{ MSE } & \multicolumn{2}{|c|}{ congruence B-loadings } & \multicolumn{2}{|c|}{ congruence C-loadings } \\
\hline & & RPARAFAC & ALS-SI & RPARAFAC & ALS-SI & RPARAFAC & ALS-SI \\
\hline$\varepsilon=0$ & $\begin{array}{c}\text { no } \\
\text { outliers }\end{array}$ & $\begin{array}{c}0.00071 \\
(1.84 \mathrm{e}-05)\end{array}$ & $\begin{array}{c}0.00071 \\
(1.84 \mathrm{e}-05)\end{array}$ & $\begin{array}{c}0.999253 \\
(2.98 \mathrm{e}-05)\end{array}$ & $\begin{array}{c}0.999370 \\
(1.95 \mathrm{e}-05)\end{array}$ & $\begin{array}{c}0.999946 \\
(3.51 \mathrm{e}-06)\end{array}$ & $\begin{array}{r}0.999949 \\
(3.35 \mathrm{e}-06)\end{array}$ \\
\hline \multirow{3}{*}{$\varepsilon=0.10$} & $\begin{array}{l}\text { residual } \\
\text { outliers }\end{array}$ & $\begin{array}{c}0.00071 \\
(1.85 \mathrm{e}-05)\end{array}$ & $\begin{array}{c}0.00216 \\
(1.28 \mathrm{e}-04)\end{array}$ & $\begin{array}{c}0.999202 \\
(3.18 \mathrm{e}-05)\end{array}$ & $\begin{array}{c}0.310238 \\
(5.59 \mathrm{e}-02)\end{array}$ & $\begin{array}{c}0.999937 \\
(3.71 \mathrm{e}-06)\end{array}$ & $\begin{array}{c}0.383883 \\
(5.22 \mathrm{e}-02)\end{array}$ \\
\hline & $\begin{array}{c}\text { good } \\
\text { leverage }\end{array}$ & $\begin{array}{c}0.00071 \\
(1.85 \mathrm{e}-05)\end{array}$ & $\begin{array}{c}0.00071 \\
(1.86 \mathrm{e}-05)\end{array}$ & $\begin{array}{c}0.999255 \\
(3.34 \mathrm{e}-05)\end{array}$ & $\begin{array}{c}0.999923 \\
(5.16 \mathrm{e}-06)\end{array}$ & $\begin{array}{c}0.999941 \\
(3.79 \mathrm{e}-06)\end{array}$ & $\begin{array}{c}0.999994 \\
(5.05 \mathrm{e}-07)\end{array}$ \\
\hline & $\begin{array}{c}\text { bad } \\
\text { leverage }\end{array}$ & $\begin{array}{c}0.00071 \\
(1.85 \mathrm{e}-05)\end{array}$ & $\begin{array}{c}0.00140 \\
(1.63 \mathrm{e}-04)\end{array}$ & $\begin{array}{c}0.999203 \\
(3.15 \mathrm{e}-05)\end{array}$ & $\begin{array}{c}0.740427 \\
(5.89 \mathrm{e}-02)\end{array}$ & $\begin{array}{l}0.999937 \\
(3.80 \mathrm{e}-06)\end{array}$ & $\begin{array}{c}0.763325 \\
(5.45 \mathrm{e}-02)\end{array}$ \\
\hline \multirow{3}{*}{$\varepsilon=0.20$} & $\begin{array}{l}\text { residual } \\
\text { outliers }\end{array}$ & $\begin{array}{c}0.00071 \\
(1.85 \mathrm{e}-05)\end{array}$ & $\begin{array}{c}0.00246 \\
(1.13 \mathrm{e}-04)\end{array}$ & $\begin{array}{c}0.999117 \\
(3.76 \mathrm{e}-05)\end{array}$ & $\begin{array}{c}0.199771 \\
(4.26 \mathrm{e}-02)\end{array}$ & $\begin{array}{c}0.999926 \\
(4.96 \mathrm{e}-06)\end{array}$ & $\begin{array}{c}0.304773 \\
(4.14 \mathrm{e}-02)\end{array}$ \\
\hline & $\begin{array}{c}\text { good } \\
\text { leverage }\end{array}$ & $\begin{array}{c}0.00071 \\
(1.85 \mathrm{e}-05)\end{array}$ & $\begin{array}{c}0.00072 \\
(1.86 \mathrm{e}-05)\end{array}$ & $\begin{array}{c}0.999288 \\
(3.68 \mathrm{e}-05)\end{array}$ & $\begin{array}{c}0.999966 \\
(1.74 \mathrm{e}-06)\end{array}$ & $\begin{array}{c}0.999940 \\
(4.97 \mathrm{e}-06)\end{array}$ & $\begin{array}{c}0.999997 \\
(1.92 \mathrm{e}-07)\end{array}$ \\
\hline & $\begin{array}{c}\text { bad } \\
\text { leverage }\end{array}$ & $\begin{array}{c}0.00071 \\
(1.85 \mathrm{e}-05)\end{array}$ & $\begin{array}{c}0.00123 \\
(1.22 \mathrm{e}-04)\end{array}$ & $\begin{array}{c}0.999117 \\
(3.76 \mathrm{e}-05)\end{array}$ & $\begin{array}{c}0.744388 \\
(5.84 \mathrm{e}-02)\end{array}$ & $\begin{array}{c}0.999926 \\
(4.96 \mathrm{e}-06)\end{array}$ & $\begin{array}{c}0.760228 \\
(5.53 \mathrm{e}-02)\end{array}$ \\
\hline
\end{tabular}




\section{List of Figures}

Figure 1: Outlier map of the Dorrit data set obtained with RPARAFAC-SI.

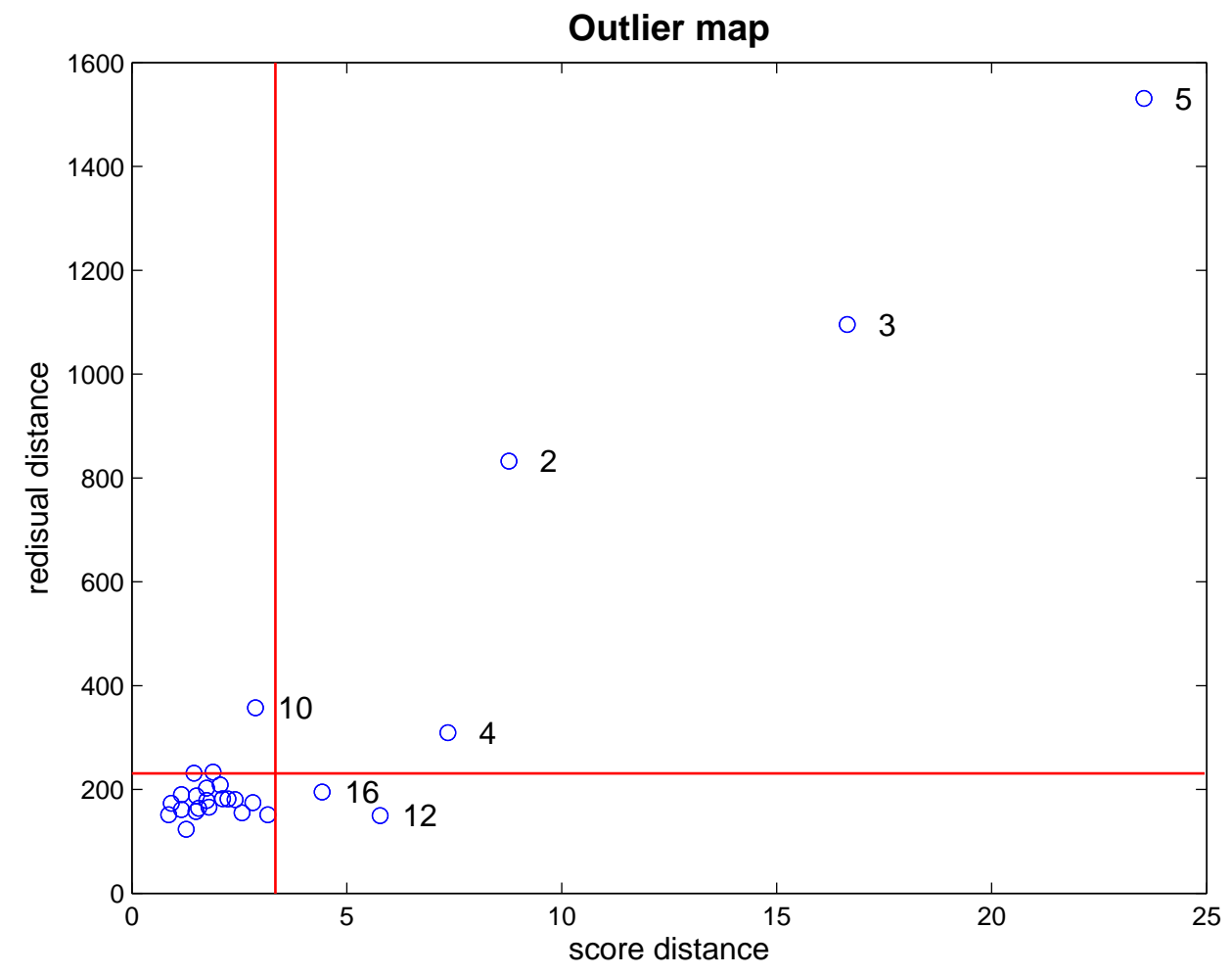


Figure 2: Loadings obtained with two robust methods. The left column shows the emission loadings (B-loadings), and the right column shows the excitation loadings (C-loadings). The top row shows the loadings for RPARAFAC-SI, the bottom row for the method of [Engelen et al., 2009].
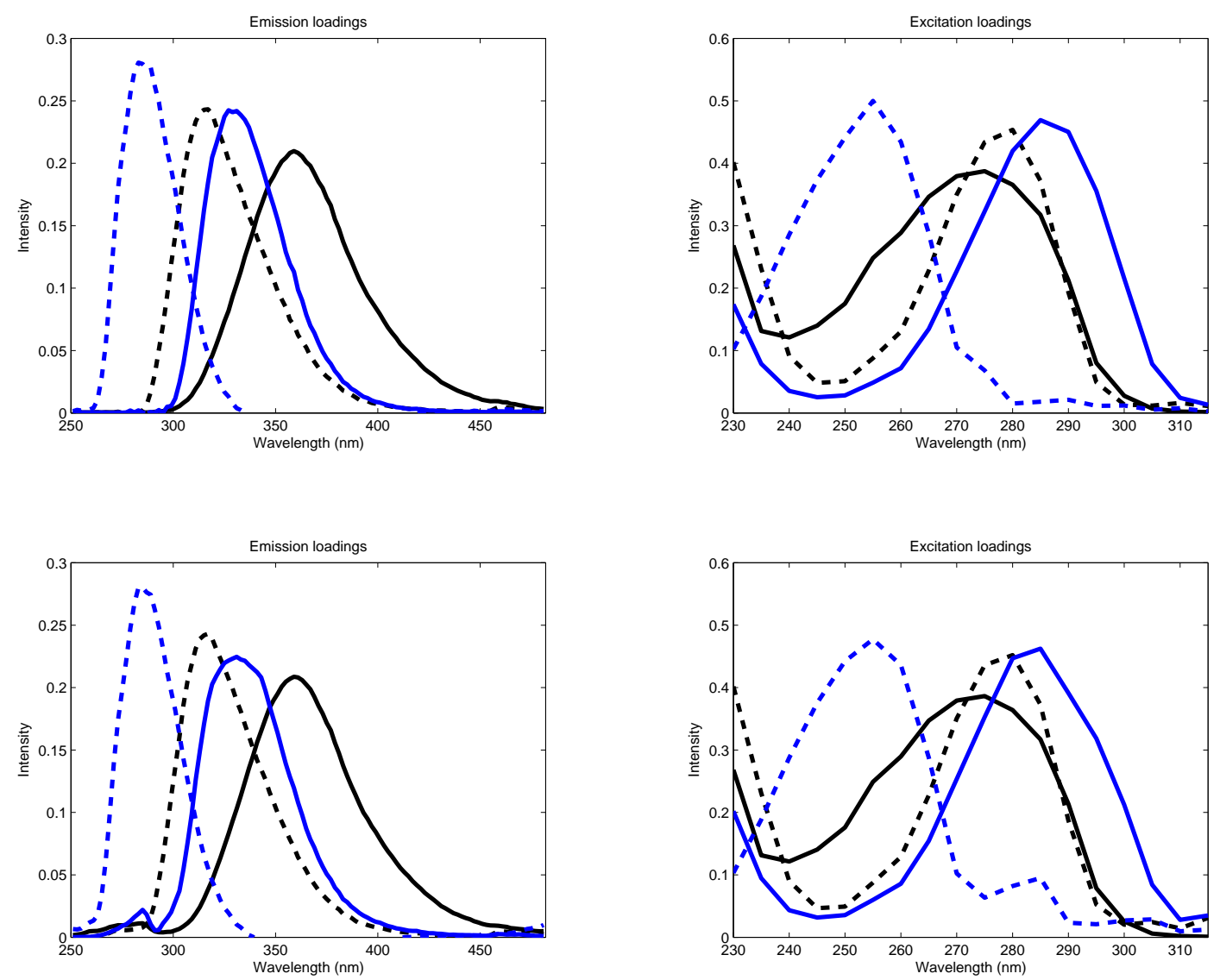
Figure 3: Plots of the estimated scores for each component of the model (vertical axis) and the true quantities of the four compounds (horizontal axis). The circles indicate the regular observations, and the crosses indicate the observations which the robust RPARAFAC-SI method flagged as outliers.
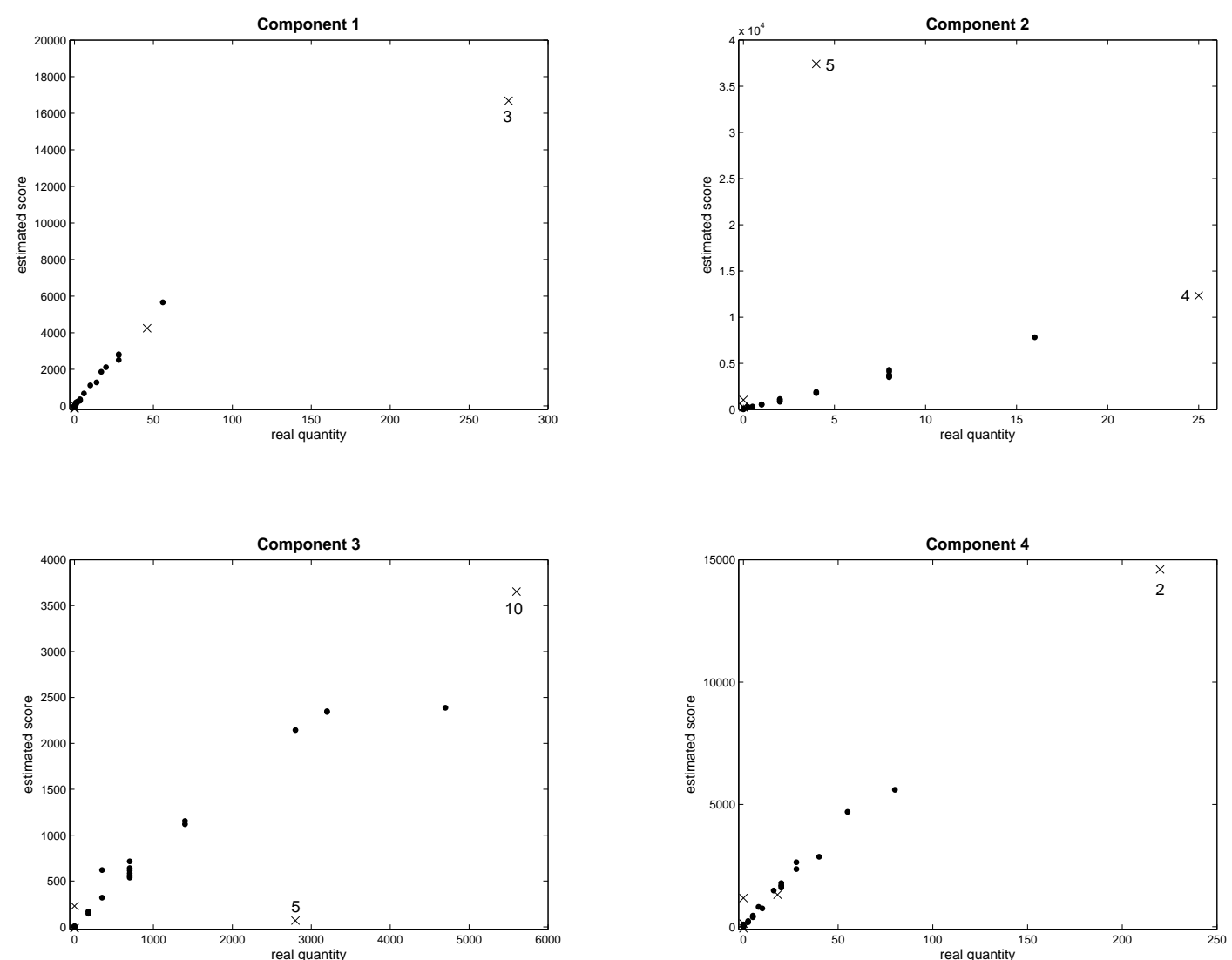